\title{
Nano-emulsion formation by emulsion phase inversion
}

\author{
Patrick Fernandez ${ }^{\mathrm{a}}$, Valérie André ${ }^{\mathrm{b}}$, Jens Rieger ${ }^{\mathrm{a}}$, Angelika Kühnle ${ }^{\mathrm{a}, *}$ \\ a BASF Aktiengesellschaft, Polymer Physics, GKP/O-G201, 67056 Ludwigshafen, Germany \\ b BASF Aktiengesellschaft, Strategic Marketing Cosmetic Ingredients, 67056 Ludwigshafen, Germany
}

Received 17 March 2004; accepted 8 September 2004

\begin{abstract}
The droplet size distribution of an emulsion governs emulsion properties such as long-term stability, texture and optical appearance. Consequently, means to control the droplet size during emulsification are of interest when well-defined emulsion properties are needed. In this work, we study emulsions consisting of water, paraffin oil and a mixture of non-ionic surfactants and fatty alcohols by means of laser light scattering. We investigate the influence of the route of preparation as well as the surfactant concentration on the droplet size distribution. Above a critical surfactant-to-oil ratio and following the standard way of emulsion phase inversion, a significant amount of oil droplets with diameters less than $1 \mu \mathrm{m}$ were obtained. When changing the way of emulsification and thereby avoiding a phase inversion to occur, such fine droplets are absent and the droplet size distribution is solely governed by the input of mechanical energy. We demonstrate that emulsification by the phase inversion method makes use of two effects for the achievement of finely dispersed oil-in-water emulsions. The lamellar or bicontinuous structure formed by the surfactant at the inversion point determines the size of the resulting droplets while the corresponding minimal interfacial tension facilitates the droplet formation, explaining why the droplet size distribution only depends on the weight ratio between surfactant and oil rather than on the water concentration.
\end{abstract}

(C) 2004 Elsevier B.V. All rights reserved.

Keywords: Emulsion; Catastrophic phase inversion; Emulsification; Phase inversion temperature (PIT); Emulsion inversion point (EIP)

\section{Introduction}

Macroemulsions are dispersions of at least two nonmiscible liquids. They are thermodynamically unstable systems that are stabilized kinetically. Consequently, the stability of an emulsion depends both on its composition and the size of the emulsion droplets. Typically, the droplet size of conventional emulsions is larger than $1 \mu \mathrm{m}$, making these droplets susceptible to gravity forces. Depending on the preparation method, different droplet size distributions might be achieved, explaining why the route of preparation can have an influence on the emulsion stability. Emulsions with droplet sizes between those of conventional emulsions and microemulsions, i.e. with a typical size range of $20-500 \mathrm{~nm}$ are termed mini-emulsions [1], ultrafine emulsions [2],

\footnotetext{
* Corresponding author. Tel.: +49 621 99176; fax: +49 62192281.

E-mail address: angelika.kuehnle@basf-ag.de (A. Kühnle).
}

submicron emulsions [3], translucent emulsions [4] and nano-emulsions [5,6]. Due to their small droplet size, nano-emulsions may appear transparent, and Brownian motion prevents sedimentation or creaming, hence offering increased stability. In contrast to microemulsions, nanoemulsions are metastable and can be diluted with water without changing the droplet size distribution [6].

The preparation of emulsions with droplet sizes in the submicrometer-range may be performed mechanically, which involves high-energy input that is generally achieved by high-shear stirring, high-pressure homogenizers, or ultrasound generators. The high-energy input leads to deforming forces that are able to break the droplets into smaller ones, provided the Laplace pressure is overcome. Additionally, an increase of the surfactant content at the interface reduces the Laplace pressure [5]. Therefore, the smaller the droplet size, the more energy and/or surfactant is required, making this preparation route unfavorable for industrial applications, 


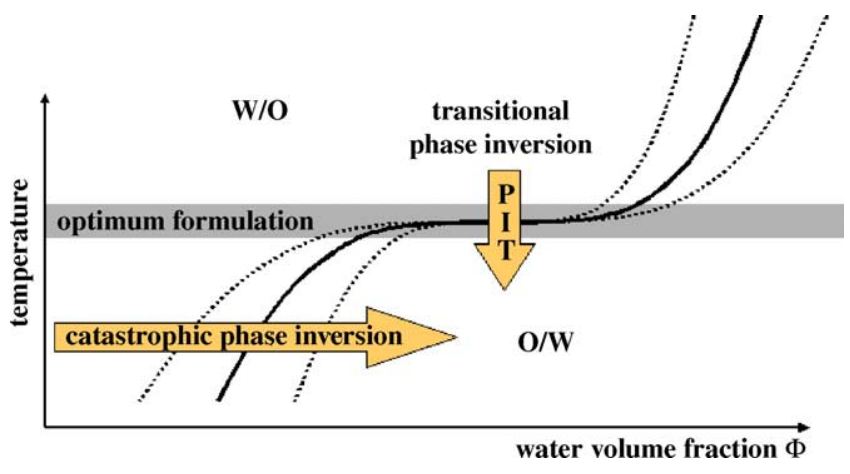

Fig. 1. Schematic illustration of both catastrophic and transitional phase inversion for the preparation of finely dispersed $\mathrm{O} / \mathrm{W}$ emulsions. The solid black line marks the inversion locus, the dotted lines the hysteresis zone. Within the optimum formulation zone and at the inversion locus, the interfacial tension is minimal. During low-energy emulsification, this ultralow interfacial tension is employed for the formation of finely dispersed droplets, while the final emulsion should be far away from these regions to enhance emulsion stability.

when very small droplets are desired. However, submicron emulsions can also be obtained by employing the physicochemical properties of the system generally referred to as low-energy emulsification methods. These methods make use of changing the spontaneous curvature of the surfactant. For non-ionic surfactants, this can be achieved by changing the temperature of the system, forcing a transition from an oil-inwater $(\mathrm{O} / \mathrm{W})$ emulsion at low temperatures to a water-in-oil (W/O) emulsion at higher temperatures (transitional phase inversion). During cooling, the system crosses a point of zero spontaneous curvature and minimal surface tension, promoting the formation of finely dispersed oil droplets (see vertical arrow in Fig. 1). This method is referred to as phase inversion temperature (PIT) method [6-9]. Instead of the temperature, other parameters such as salt concentration or $\mathrm{pH}$ value may be considered as well, generalized by considering the surfactant affinity difference (SAD) instead of the temperature alone [10].

Additionally, a transition in the spontaneous radius of curvature can be obtained by changing the water volume fraction (emulsion inversion point (EIP) method). By successively adding water into oil, initially water droplets are formed in a continuous oil phase. Increasing the water volume fraction changes the spontaneous curvature of the surfactant from initially stabilizing a W/O emulsion to an $\mathrm{O} / \mathrm{W}$ emulsion at the inversion locus. This process is well known for short-chain surfactants which form flexible monolayers at the oil-water interface, resulting in a bicontinuous microemulsion at the inversion point. Also during this transition-referred to as catastrophic phase inversionminimal interfacial tension are achieved and reported to facilitate the formation of fine droplets (horizontal arrow in Fig. 1) [10-13].

In addition to the low interfacial tension, the formation of a bicontinuous or lamellar structure with a characteristic, surfactant-dependent repeat distance at the point of phase inversion has been mentioned to play a role for the formation of submicrometer-sized droplets [14].

In the present work, the preparation of $\mathrm{O} / \mathrm{W}$ emulsions stabilized by a mixture of two non-ionic surfactants commercially used for the formulation of creams and lotions was studied in the light of the low-energy emulsification methods discussed above. We demonstrate that also for these long-chain surfactants phase inversion and nano-emulsion formation occur. The influence of the preparation route was investigated by analyzing the droplet size distribution of oil-in-water emulsions obtained by pouring the water phase into the oil and vice versa. We observed that a critical amount of surfactant is needed for the preparation of submicrometersized droplets by the emulsion inversion point method. Furthermore, we studied three surfactant-to-oil weight ratios and successively added water, thereby mapping parts of the surfactant/oil/water phase diagram. The final droplet size does not depend on the amount of water added to the system, demonstrating that the droplet size is determined by the surfactant-to-oil weight ratio rather than by the water content. This suggests that the droplet size depends on the distance given by the lamellar structure at the inversion point.

\section{Material and methods}

\subsection{Chemicals}

Emulsions were prepared with Cremophor ${ }^{\circledR}$ A6 and Cremophor $^{\circledR}$ A25 (both BASF products), distilled water and paraffin oil (mineral oil, Riedel de Haën, Germany). Cremophor ${ }^{\circledR}$ A6 is a non-ionic surfactant consisting of a polyethylene glycol alkyl ether (ceteareth- $6 ; \mathrm{C}_{i} \mathrm{E}_{j}$ with $i=16-18$ and $j=6$ ) and stearyl alcohol at a weight ratio 3:1. Cremophor ${ }^{\circledR} \mathrm{A} 25$ is also known as ceteareth-25 $\left(\mathrm{C}_{i} \mathrm{E}_{j}\right.$ with $i=16-18$ and $j=25$ ). The weight ratio Cremophor ${ }^{\circledR} \mathrm{A} 6 / \mathrm{A} 25$ was fixed at 7:3. Each sample (with compositions as indicated in Table 1) was prepared with a total mass of $300 \mathrm{~g}$, and the water-to-oil weight ratio was kept constant at 3.5. Previous to emulsification, the surfactants were mixed into the oil phase at $80^{\circ} \mathrm{C}$. The water (also at $80^{\circ} \mathrm{C}$ ) and oil phases were mixed with a Heidolph IKA stirrer at $150 \mathrm{rpm}$ and subsequently homogenized with a T25 Ultra-Turrax at 13,000 rpm for $1 \mathrm{~min}$. The samples were cooled at room temperature under moderate stirring at $150 \mathrm{rpm}$.

Table 1

Water/Cremophor ${ }^{\circledR}$ A6/A25/paraffin oil emulsions compositions

\begin{tabular}{llll}
\hline$W_{\text {water }}$ & $W_{\text {Cremophor }}$ & $W_{\text {paraffin oil }}$ & $W_{\text {Cremophor }} / W_{\text {paraffin oil }}$ \\
\hline 75.8 & 2.5 & 21.7 & 0.12 \\
73.9 & 5 & 21.1 & 0.24 \\
71.9 & 7.5 & 20.6 & 0.36 \\
70.0 & 10 & 20 & 0.5 \\
\hline$W_{\text {water }}, W_{\text {Cremophor }}$ and $W_{\text {paraffin oil }}$ are the weight fractions (in wt. $\%$ ) of water, \\
Cremophor \\
indicates the surfactant-to-oil weight ratio.
\end{tabular}


For all compositions indicated in Table 1, the water phase was poured into the oil phase (described as $\mathrm{W}$ into $\mathrm{O}$ or method A). Furthermore, an additional sample containing $10 \%$ Cremophor $^{\circledR}$ mixture was prepared by pouring the oil phase into the water phase $(\mathrm{O}$ into $\mathrm{W}$ or method B).

For the mapping of the phase diagram, increasing amounts of water were added to mixtures of surfactant and oil at $80^{\circ} \mathrm{C}$. The total sample mass was $25 \mathrm{~g}$. The surfactant-to-oil weight ratios correspond to those listed in Table 1 . After weighing, each sample was shaken by hand, and the samples were stored in a thermostatized chamber at $80^{\circ} \mathrm{C}$ for several days in order to equilibrate the samples.

\subsection{Methods}

The oil droplet size distribution was measured by means of laser light scattering (HORIBA La-900). A few drops of emulsion were injected into a bath of distilled water kept at $25^{\circ} \mathrm{C}$ (sample dilution). The bath is continuously under agitation in order to disperse the oil droplets within the mixture. A few minutes after pouring, the laser light scattering measurement was performed.

\section{Results}

The resulting droplet size distributions for the emulsions listed in Table 1 are given in Fig. 2. Depending on the surfactant concentration, the droplet sizes vary when prepared by method A. For $2.5 \mathrm{wt} . \%$ surfactant (surfactant-to-oil weight ratio of $\sim 0.12$ ), one large peak centered around $10 \mu \mathrm{m}$ with a small additional peak around $0.6 \mu \mathrm{m}$ is obtained. When increasing the surfactant content to $5 \mathrm{wt} . \%$ (surfactant-to-oil weight ratio of $\sim 0.24$ ), a truly bimodal distribution is observed with one peak around $8-10 \mu \mathrm{m}$ and a second centered at $\sim 0.6 \mu \mathrm{m}$. Further increasing the surfactant concentration to $7.5 \mathrm{wt} . \%$ results in one peak at $0.4 \mu \mathrm{m}$, while for the $10 \mathrm{wt} . \%$ Cremophor $^{\circledR}$ mixture one peak at around $0.3 \mu \mathrm{m}$ is obtained (surfactant-to-oil weight ratios of $\sim 0.36$ and 0.5 , respectively). Interestingly, when prepared by method B, the emulsion with $10 \mathrm{wt} . \%$ surfactant mixture shows large droplets similar to the situation observed with $2.5 \mathrm{wt} . \%$ surfactant prepared by method A. As shown in Fig. 2e, a bimodal distribution is obtained with a large peak around $10 \mu \mathrm{m}$ and a small one at $0.6 \mu \mathrm{m}$.

As shown in Fig. 3, parts of the phase diagram were mapped by diluting samples with fixed surfactant-to-oil ratios $(0.50,0.36$ and 0.12 marked by $1-3$, respectively) with water and moderately shaking by hand. Upon dilution with water, the emulsion inversion point is reached and an oil-inwater emulsion is formed. As indicated in the phase diagram, the emulsion inversion point was crossed at a water content between $20 \mathrm{wt} . \%$ (for a surfactant-to-oil weight ratio of 0.12 ) and $25 \mathrm{wt} . \%$ (for a surfactant-to-oil weight ratio of 0.50 ). The resulting emulsion was examined between crossed polarizing filters, revealing birefringence for the obtained emulsions.

For the dilution lines marked by 1 and 2, corresponding to a surfactant-to-oil weight ratio of 0.50 and 0.36 , all droplets observed were in the submicrometer-size range. As given in Table 2, the measured droplet size does not significantly change upon dilution with water. Laser light scattering revealed similar droplet size distributions, in location (centered around $0.5-0.3 \mu \mathrm{m}$ ), as well as in shape (monomodal with similar degree of polydispersity) to those obtained with the final emulsions.

\section{Discussion}

For the emulsions prepared by method A, it appears that the higher the surfactant concentration, the smaller the droplets that can be obtained. A gap exists between droplets with sizes centered at $\sim 8-10$ and $0.3-0.6 \mu \mathrm{m}$ rather than a continuous change in droplet size. This suggests two independent mechanisms of emulsification. The small-sized droplets do not originate from the mechanical energy input, since the smallest droplet size obtained by means of Ultra-Turrax homogenization is known to be around $1 \mu \mathrm{m}$ $[3,5]$. On the other hand, the droplet size centered around $8-10 \mu \mathrm{m}$ seems to agree with the mechanical disruption of the oil droplets (and possible partial coalescence of those oil droplets) and is therefore attributed to the homogenization process.

For high surfactant concentrations, however, the mechanical emulsification becomes insignificant for emulsions prepared by method A, since only oil droplets with sizes around $0.4 \mu \mathrm{m}$ (7.5 wt.\% Cremophor ${ }^{\circledR}$ mixtures) are observed. This size is further reduced to $0.3 \mu \mathrm{m}$ for $10 \mathrm{wt} . \%$ surfactant, indicating that high surfactant contents promote the nonmechanical emulsification process.

In contrast to the above observations, the sample containing $10 \mathrm{wt} . \%$ surfactant prepared by method B shows a distribution of droplets mainly centered at $8-10 \mu \mathrm{m}$, that can be attributed to the mechanical process alone. This indicates that the order of adding the phases (water poured into the oil phase or vice versa) plays a key role in the formation of small-sized oil droplets. Note that the case of emulsification by the PIT method is excluded, since this effect is independent of the order of adding the phases. Additionally, in the present experiments, all phases were subjected to the same heating and cooling procedure. Furthermore, the PIT of the Cremophor ${ }^{\circledR}$ A6/A25 surfactant mixture is above $100^{\circ} \mathrm{C} .{ }^{1}$ Therefore, the change in the water volume fraction is assumed to be responsible for the non-mechanical emulsification, leading to

\footnotetext{
${ }^{1}$ The PIT can be obtained by measuring the change in conductivity of an $\mathrm{O} / \mathrm{W}$ emulsion upon heating. At the inversion point, a non-conductive $\mathrm{W} / \mathrm{O}$ emulsion is formed, resulting in a sharp decrease in conductivity. For the surfactant mixture investigated here, no such drop could be observed up to a temperature of about $90^{\circ} \mathrm{C}$.
} 

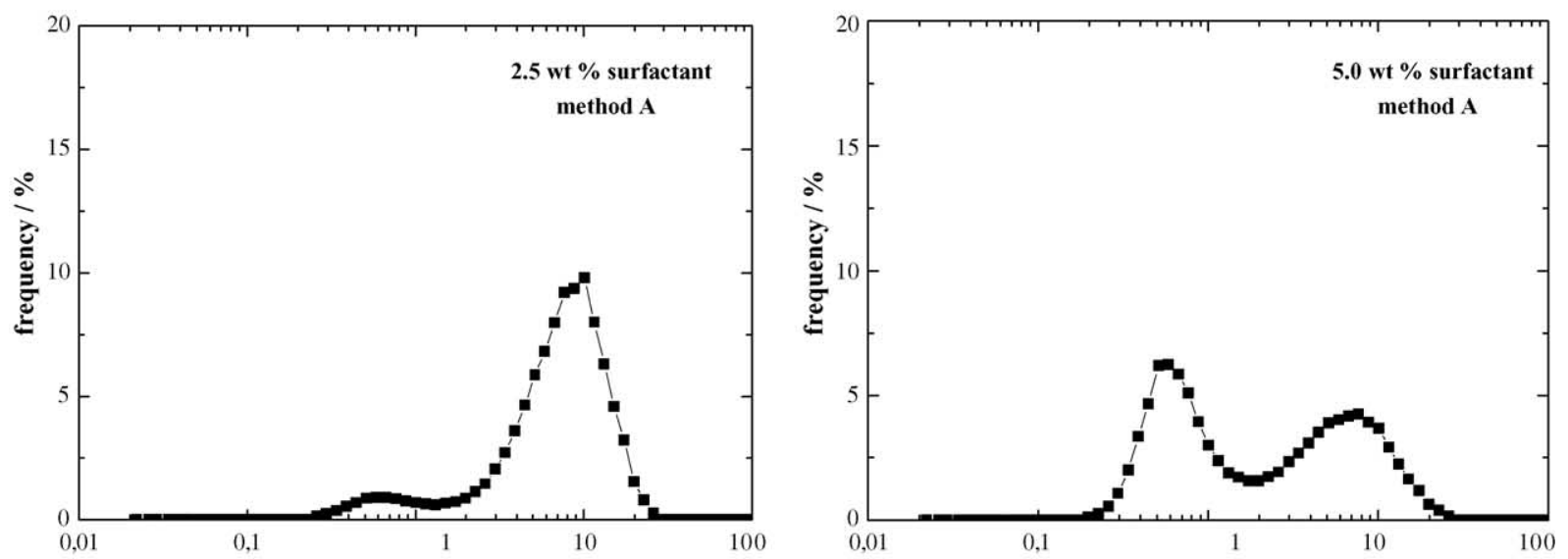

(a)

diameter $/ \mu \mathbf{m}$

(b)

diameter $/ \mu \mathbf{m}$
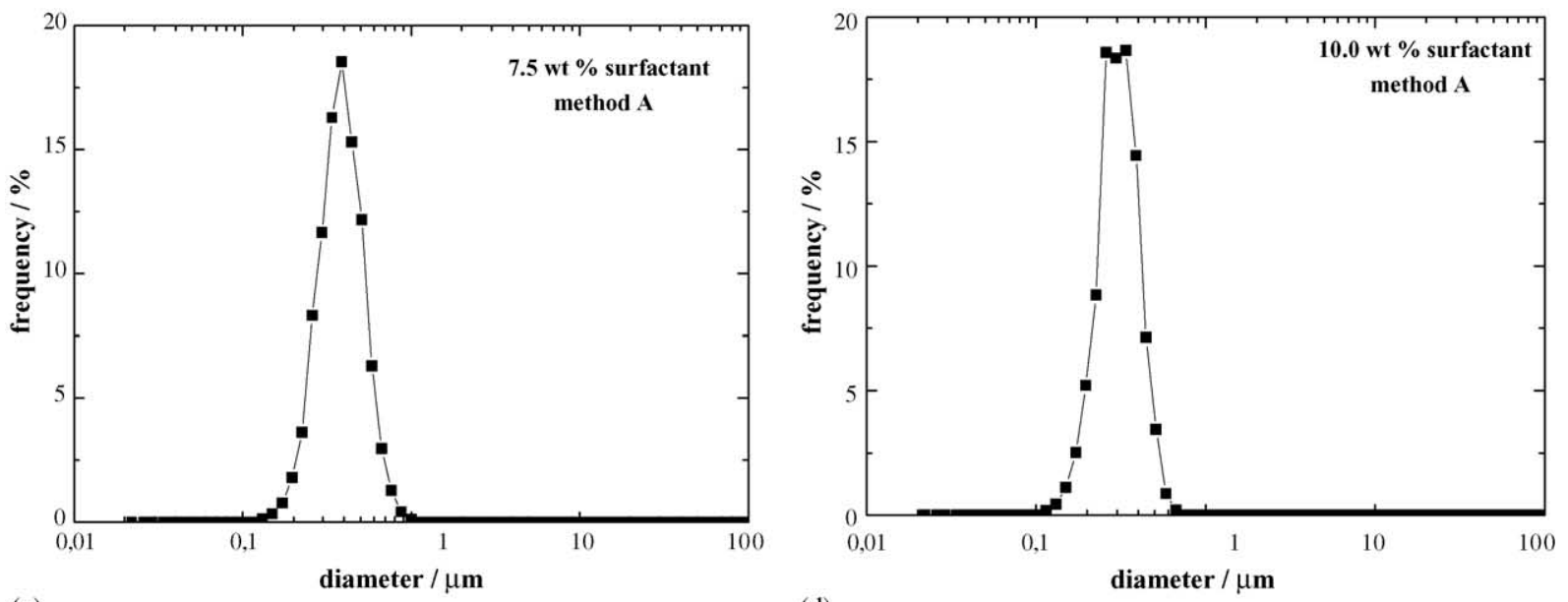

(c)

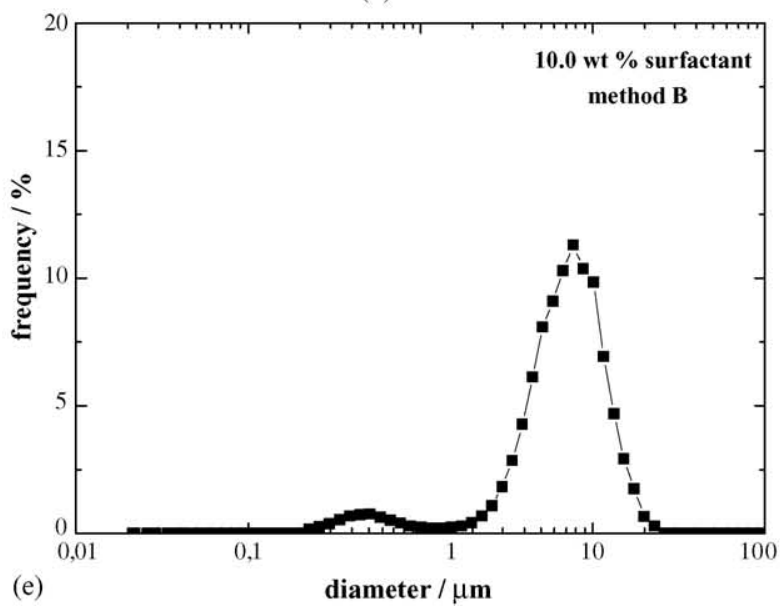

Fig. 2. Droplet size distributions depending on the surfactant concentration ((a) $2.5 \mathrm{wt} . \%$; (b) $5 \mathrm{wt} . \%$; (c) $7.5 \mathrm{wt} . \%$; (d) $10 \mathrm{wt} . \%$; (e) $10 \mathrm{wt} . \%$ ) and the preparation method ((a)-(d) according to method A; (e) according to method B).

submicrometer-sized oil droplets obtained by means of emulsion phase inversion.

A comparison of Fig. 2 and Table 2 demonstrates that no difference exists between the droplet size distributions of emulsions obtained by means of Ultra-Turrax and by manual shaking for surfactant-to-oil weight ratios of 0.36 and 0.50. This further emphasizes the fact that the non- mechanical emulsification is a spontaneous process and already gentle mixing is sufficient to allow for the formation of submicrometer-sized droplets.

As can be seen in Fig. 2, a critical surfactant-to-oil weight ratio (larger than 0.24 ) is needed to exclusively obtain submicrometer-sized droplets. On the other hand, upon addition of water, the droplet size distribution does not change 


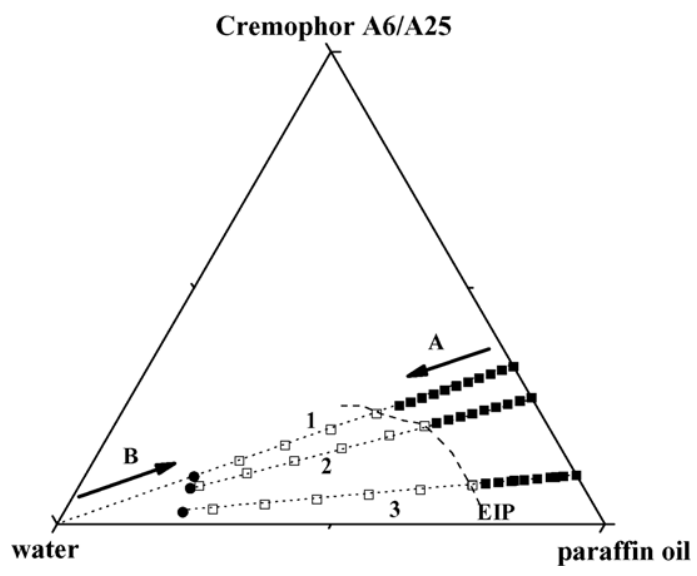

Fig. 3. Presentation of the different emulsification procedures in the phase diagram of water/Cremophor ${ }^{\circledR}$ mixture/paraffin oil at $80^{\circ} \mathrm{C}$ (in weight fractions). The two different emulsification methods, water phase poured into the oil phase (A) and vice versa (B) are given by the arrows. The dashed line marks the emulsion inversion point (EIP). Dotted lines indicate dilution, leading to final emulsions (closed circles) with 10, 7.5, and $2.5 \mathrm{wt} . \%$ surfactant mixture, respectively (see Table 1). Open squares show samples for which oil-in-water emulsions were observed, while closed squares indicate compositions for which the phase separation has no occurred yet.

significantly once the EIP is crossed (Fig. 3 and Table 2), suggesting that for spontaneous emulsification by phase inversion the surfactant-to-oil weight ratio is of importance rather than the amount of water.

Our results suggest that the droplet size distribution is mainly controlled by the structure of the bicontinuous or lamellar phase formed during the phase inversion, the characteristic distance of which is dependent on the surfactant-to-oil ratio. This is in agreement with literature data [6] showing that for emulsions produced by the PIT method, the droplet
Table 2

Droplet sizes of emulsions with surfactant-to-oil ratios of 0.36 and 0.50 , respectively, upon dilution with water

\begin{tabular}{lllll}
\hline$W_{\text {paraffin oil }}$ & $W_{\text {Cremophor }}$ & $W_{\text {water }}$ & $\begin{array}{l}W_{\text {Cremophor }} / \\
W_{\text {paraffin oil }}\end{array}$ & $\begin{array}{l}\text { Droplet size } \\
(\mu \mathrm{m})\end{array}$ \\
\hline 51.3 & 18.7 & 30 & 0.364 & 0.5 \\
44 & 16 & 40 & 0.364 & 0.33 \\
36.7 & 13.3 & 50 & 0.364 & 0.35 \\
29.3 & 10.7 & 60 & 0.364 & 0.35 \\
22 & 8 & 70 & 0.364 & 0.36 \\
40 & 20 & 40 & 0.5 & 0.36 \\
33.33 & 16.67 & 50 & 0.5 & 0.38 \\
26.67 & 13.33 & 60 & 0.5 & 0.33 \\
20 & 10 & 70 & 0.5 & 0.38 \\
\hline
\end{tabular}

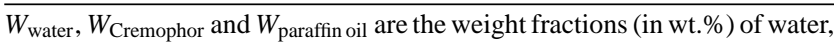
Cremophor ${ }^{\circledR}$ mixture and paraffin oil, respectively. $W_{\text {Cremophor }} / W_{\text {paraffin oil }}$ indicates the surfactant-to-oil weight ratio. All particle size distributions are monomodal and centered at the given droplet size.

size is governed by the structure of the bicontinuous phase, rather than the water content. The high surfactant-to-oil ratio used in this work results in a lamellar phase with a repeat distance of some few tens of nanometer. The observed droplets are, however, in the range of 300-500 $\mathrm{nm}$. This discrepancy suggests that the droplets grow after formation by Ostwald ripening similar to what has been reported upon before [14]. Furthermore, this consideration suggests that the low interfacial tension associated with the inversion point, which is usually assumed to assist the droplet formation, might be of secondary importance.

In order to illustrate the mechanisms involved in this lowenergy emulsification process, the steps of submicron-droplet formation by phase inversion are given in the following. As the water phase is poured into the oil phase, the system starts as a W/O microemulsion [15]. Upon increasing the volume
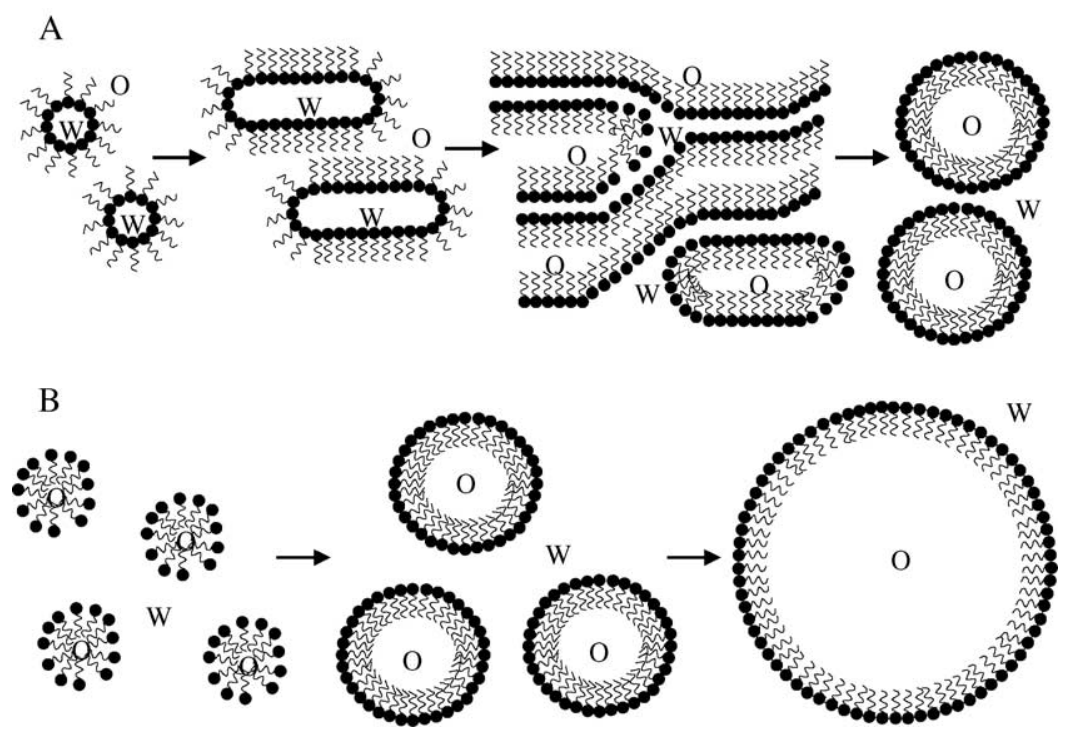

Fig. 4. Scheme of the emulsification procedure (W: water phase; O: oil phase). Method A: first water-in-oil droplets are produced; those inverted structures merge together to give elongated and bicontinuous or lamellar structures that finally decompose into submicrometer-sized oil droplets. Method B: small oil droplets are immediately produced and grow in size upon oil and surfactant addition. Their size is a result of the mechanical process only. 
fraction of water, water droplets merge together and bicontinuous or lamellar [15] structures are formed, which, after the EIP is passed, decompose into smaller oil droplets upon further increasing the water content. Further dilution with water does not change the droplet size at this stage of droplet formation. The decomposition into very finely dispersed droplets is facilitated by the fact that the interfacial tension is minimal at the EIP. A high surfactant concentration allows for a complete solubilization of the oil near the EIP [6], leading to monomodal emulsions with submicrometer-sized droplets. For low or medium surfactant concentrations, the oil solubilization is not complete, resulting in larger droplets that arise from mechanical emulsification. A critical surfactant concentration is therefore required to guarantee bicontinuous or lamellar structures, which in turn decompose into submicrometer-sized oil droplets after phase inversion. Considering the theoretical amount of surfactant required for stabilization of the final droplets, an estimated value of about $10 \%$ for the surfactant-to-oil ratio is obtained. ${ }^{2}$ This suggests that the surfactant used in our study is in excess to what is needed to stabilize the droplets. The fact that the final emulsions are birefringent indicates the existence of a liquid crystalline structure in the emulsions. We therefore suggest that a large amount of the surfactant is consumed in the external phase forming this crystalline structure. Small angle X-ray scattering (SAXS) experiments performed on this sample also affirm the existence of a crystalline structure [16]. This excess surfactant might, however, still contribute to the emulsion stability by increasing the viscosity of the external phase.

If the oil phase is added to the water phase (method B), no such transition through bicontinuous or lamellar structures (those structures remain to be better defined) occurs since a critical volume fraction of the oil phase cannot be reached. The two procedures are exemplified in Fig. 4.

\section{Conclusion}

The present results demonstrate the importance of the way of emulsification on the droplet size distribution. When emulsifying via emulsion phase inversion, finely dispersed oil droplets can be achieved, much smaller than by mechan- ical emulsification solely. We demonstrate that a critical surfactant concentration is necessary for emulsification via the EIP method. While low interfacial tension might facilitate the droplet formation, the resulting droplet size distribution mainly depends on the surfactant-to-oil ratio, suggesting that the size of the droplets is governed by the lamellar or bicontinuous structure formed at the inversion point.

\section{Acknowledgement}

The authors thank H. Debus for her support when using the laser light scattering apparatus.

\section{References}

[1] M. El-Aasser, C. Lack, J. Vanderhoff, F. Fowkes, Colloid Surf. 29 (1988) 103.

[2] H. Nakajima, Industrial Applications of Microemulsions, Marcel Dekker, New York, 1997.

[3] S. Benita, M.Y. Levy, J. Pharm. Sci. 82 (1993) 1069.

[4] A.J.F. Sing, A. Gracia, J. Lachaise, P. Brochette, J.L. Salager, Colloid Surf. A 152 (1999) 31.

[5] C. Solans, J. Esquena, A.M. Forgiarini, N. Ulson, D. Morales, P. Izquierdo, N. Azemar, M.J. Garcia-Celma, Absorption and Aggregation of Surfactants in Solution, vol. 109, Marcel Dekker, New York, 2003.

[6] D. Morales, J.M. Gutiérrez, M.J. García-Celma, C. Solans, Langmuir 19 (2003) 7196.

[7] K. Shinoda, H.J. Saito, Colloid Interface Sci. 30 (1969) 258.

[8] T. Engels, W. Förster, W. von Rybinski, Colloid Surf. A 99 (1995) 141.

[9] F. Shambil, World Patent WO 89/11907.

[10] J.L. Salager, Encyclopedia of Emulsion Technology, vol. 3, Marcel Dekker, New York, 1988.

[11] J.L. Salager, L. Marquez, I. Mira, A. Pena, E. Tyrode, N.B. Zambrano, Absorption and Aggregation of Surfactants in Solution, vol. 109, Marcel Dekker, New York, 2003.

[12] B.W. Brooks, H.N. Richmond, M. Zerfa, Modern Aspects of Emulsion Science, Cambridge, 1998, pp. 175-204.

[13] F. Bouchama, G.A. van Aken, A.J.E. Autin, G.J.M. Koper, Colloid Surf. A 231 (2003) 11.

[14] L. Taisne, B. Cabane, Langmuir 14 (1998) 4744.

[15] A. Forgiarini, J. Esquena, C. Gonzalez, C. Solans, Langmuir 17 (2001) 2076.

[16] P. Fernandez, L. Börger, Y. Men, J. Rieger, V. André, A. Kühnle, in preparation.

\footnotetext{
${ }^{2}$ With a surfactant layer thickness of about $L=5 \mathrm{~nm}$ and a droplet size of $300 \mathrm{~nm}$, one obtains a surfactant-to-oil volume ratio of $\frac{4 \pi R^{2} L}{\frac{4}{3} \pi R^{3}}=\frac{3 L}{R}$ $=\frac{3 \times 5 \mathrm{~nm}}{150 \mathrm{~nm}}=0.1$
} 